\title{
The Application of Numerical Manifold Method to the Turning Point Singularity Problem in Seepage
}

\author{
Qiang Feng 1,2,a, Weipeng Zhang 1,2,b
}

${ }^{1}$ Institute of Rock and Soil Mechanics, Chinese Academy of Sciences, Wuhan, Hubei 430071

2 University of Chinese Academy of Sciences, Beijing 100049, China

a qiangf2000@163.com, b zhangweipeng@qq.com

Keywords: numerical manifold method, seepage, turning point singularity

Abstract: The numerical manifold method with special physical patch is used to deal with the seepage problem of turning point singularity caused by sheet pile. The method is applied to four engineering examples, and is superior to the finite element method, when the results are compared with the general finite element method.

\section{Introduction}

Numerical Manifold method(NMM)[1] consists of three parts: (1) Mathematical Cover and Physical Cover, which allows the interpolation grid and the integral grid to be separated to facilitate the processing of strong discontinuities problem; (2) Numerical Manifold Method Space, numerical manifold method extends the finite element space, and a function space can be defined on each physical piece; (3) Unit decomposition function. Therefore, numerical manifold method is easy to deal with seepage problem[2-5], and can construct local special physical patch at singular point[4].

\section{The Singular Physical Patch of NMM}

This is physical patch of singularity point:

$$
\begin{aligned}
P_{\text {Singular }}^{T} & =\left[p_{1}, p_{2}\right] \\
d_{\text {Singular }}^{T} & =\left[d_{1}, d_{2}\right] \\
\phi_{\text {Singular }} & =P_{\text {Singular }}^{T} \cdot d_{\text {Singular }}
\end{aligned}
$$

where,

$$
\begin{gathered}
p_{1}=1 \\
p_{2}=r^{\frac{\pi}{2 \varphi}} \sin \left(\frac{\pi \theta}{2 \varphi}\right)
\end{gathered}
$$

$p_{1} 、 p_{2}$ are basis function, $d$ is a generalized degree of freedom, $(r, \theta)$ is the polar coordinate, and the pole is the singular corner, $\varphi$ is half of the reentrant corner of the singular point.

Using the unit decomposition property of the shape function, the head function $\phi^{e}$ (potential function) at any point in the local unit satisfies:

$$
\phi^{e}(x, y)=\sum_{i=1}^{n} N_{i} \phi_{i}=\sum_{i=1}^{n} N_{i}(x, y) \sum_{k=1}^{m} p_{k}(x, y) d_{i k}=\sum_{j=1}^{n m} T_{j} d_{j}
$$

where, $\phi_{i}$ is the head function covered by the manifold element, $N_{i}$ is the unit shape function, $p_{k}$ is the basis function, $T_{j}=N_{i} p_{k}$ is the generalized shape function, $d_{j}=d_{i k}$ is the generalized degree of freedom. The rest according to the general NMM processes, not repeat the details[2-3].

\section{Engineering Application Examples}

3.1 Example One. There is a weir problem with sheet piles[7] in Fig.1,A is singulary point.Flow net shown in Fig. 2 to Fig.3, which is calculated separately by NMM and finite element method(FEM). 


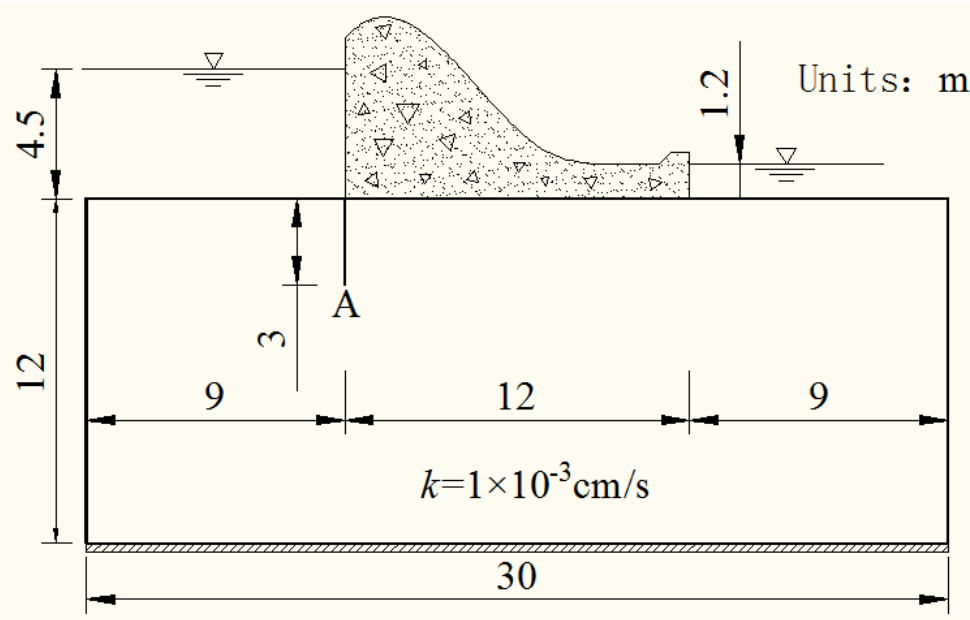

Fig.1 Example one Weir problem with sheet piles A

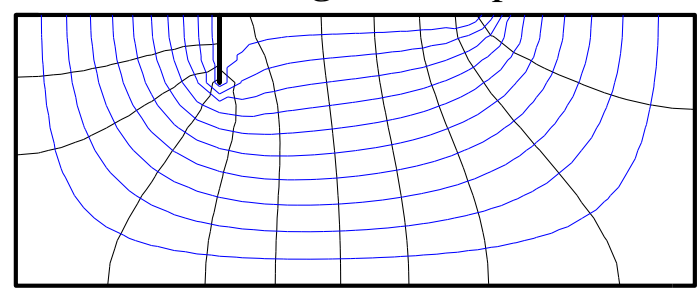

Fig.2 Flow net with NMM of example one

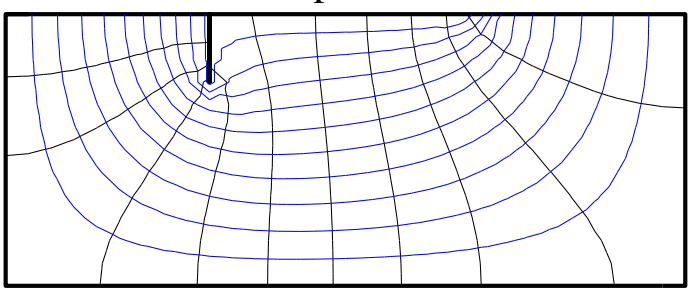

Fig.3 Flow net with FEM of example one

3.2 Example Two. There is a weir problem with sheet piles in Fig.4, D is singulary point.Flow net shown in Fig.5 to Fig.6, which is calculated separately by NMM and FEM.

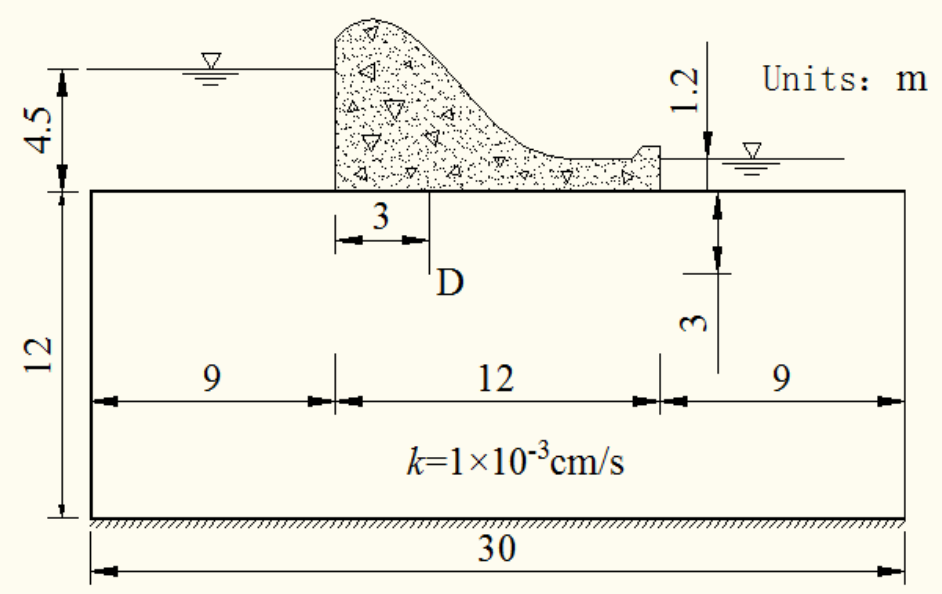

Fig.4 Example two Weir problem with sheet piles D

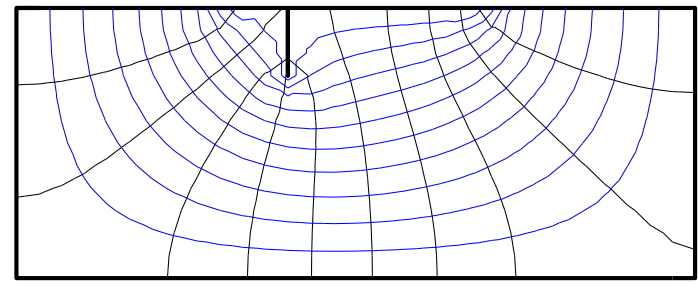

Fig.5 Flow net with NMM of example two

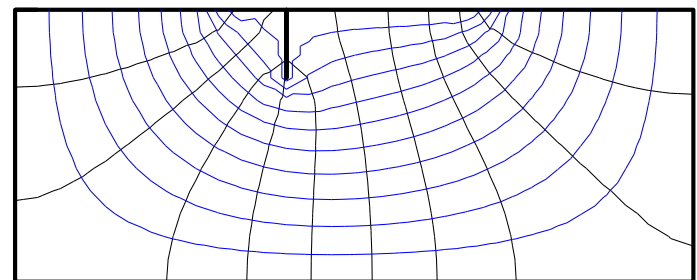

Fig.6 Flow net with FEM of example two

3.3 Example Three. There is a weir problem with sheet piles in Fig.7, E is singulary point.Flow net shown in Fig.8 to Fig.9, which is calculated separately by NMM and FEM. 


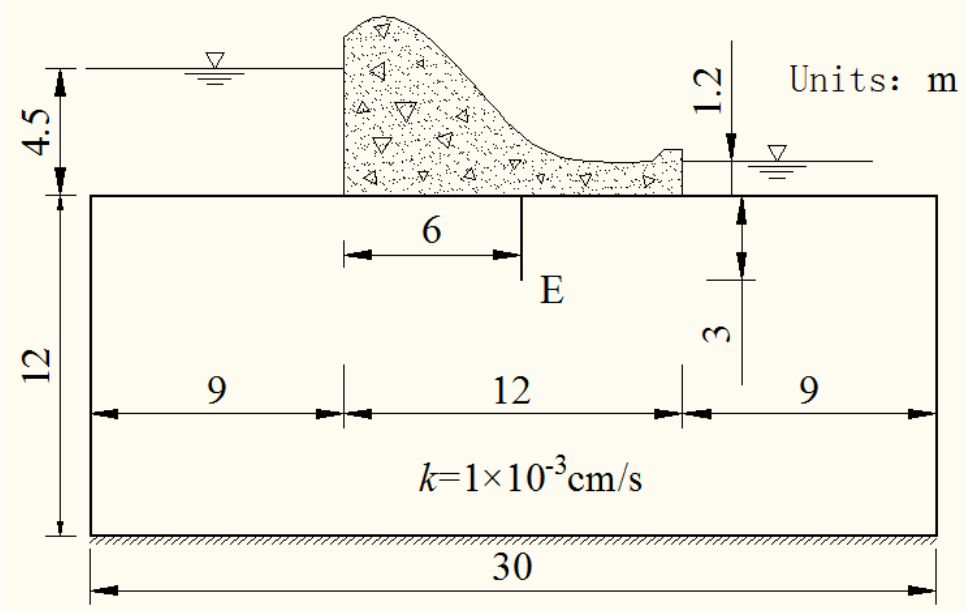

Fig.7 Example three Weir problem with sheet piles E

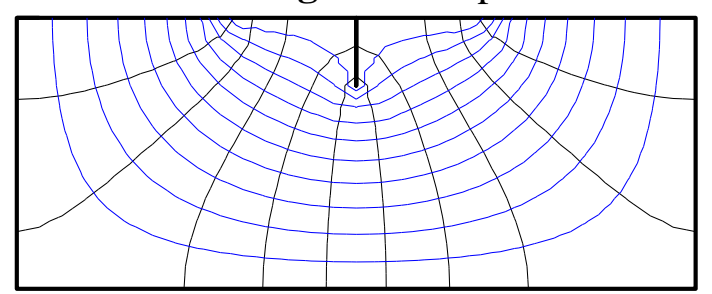

Fig.8 Flow net with NMM of example three

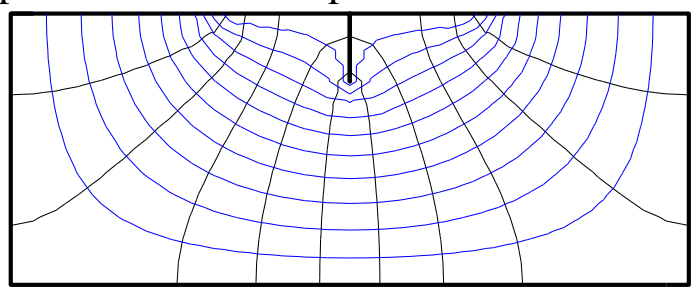

Fig.9 Flow net with FEM of example three

3.4 Example Four. There is a weir problem with sheet piles in Fig.10, B is singulary point.Flow net shown in Fig.11 to Fig.12, which is calculated separately by NMM and FEM.

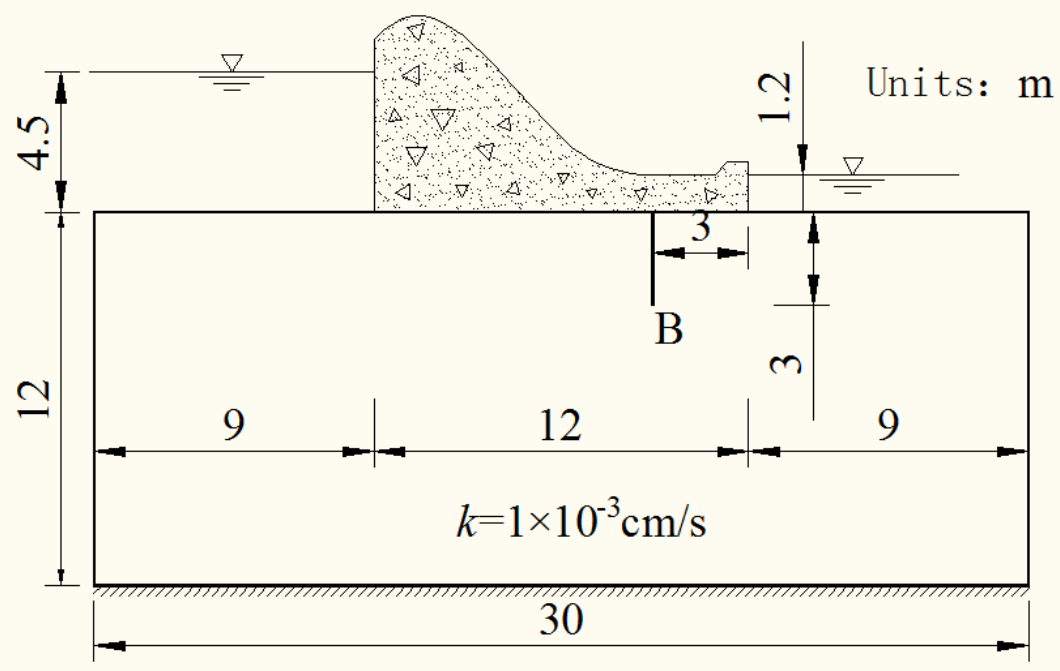

Fig.10 Example four Weir problem with sheet piles B

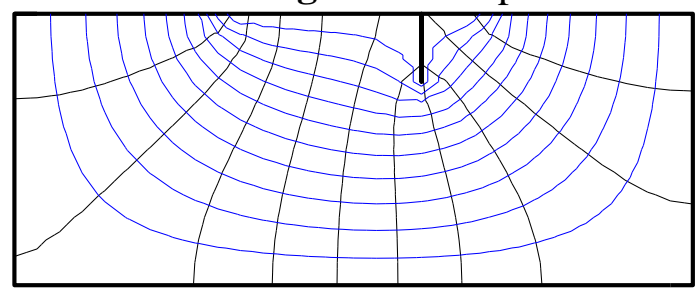

Fig.11 Flow net with NMM of example four

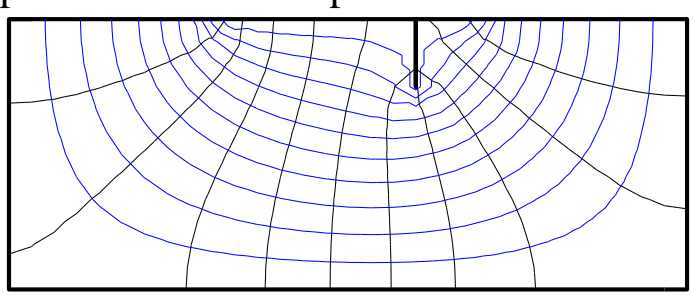

Fig.12 Flow net with FEM of example four

\section{Conclusions}

Sum up the above analysises, The streamlines calculated by NMM rise slowly near the singularity, while the streamlines that are computed by FEM rise steep near the singularity. This is because FEM 
does not consider singularity. The singularity here means that the potential function is continuous and the partial derivative is discontinuous. And the equipotential lines calculated by the manifolds are smoother.

NMM is more suitable for solving seepage problems, especially for some local cases where there are special solutions. NMM can construct special local physical patch function.

\section{Acknowledgements}

This work was financially supported by the National Natural Science Founds of China (11172313).

\section{References}

[1] Shi, G.H., Manifold method of material analysis. Trans.army Conf.on Appl.math. \& Comp.u.s.army Res.office, 1992.

[2] FENG Qiang, Numerical Manifold Method and Its Application in unconfined Seepage.2005,China Three Gorges University.In Chinese.

[3] JIANG Qing-hui, DENG Shu-shen, ZHOU Chuang-bing, Three-dimensional numerical manifold method for seepage problems with free surfaces, Rock and Soil Mechanics, 2011. 32(3): p. 879-884.In Chinese.

[4] Aalto, J., Singularity Elements for Seepage Analysis. International Journal for Numerical and Analytical Methods in Geomechanics, 1985. 9(2): p. 185-196.

[5] Harr, M.E., Groundwater and seepage. 1962: McGraw-Hill. iv.

[6] Yuan Lianju, Engineering seepage mechanics and its application. 2001: China Building Materials Industry Press.In Chinese.

[7] TRACY F T, RADHAKRISHNAN N. Automatic Generation of Seepage Flow Nets by Finite Element Method [J]. Journal of Computing in Civil Engineering, 1989, 3(3): 268-84. 